\title{
LA IMAGEN DE LA SPHAERA INFINITA DEL ANÓNIMO LIBER XXIV PHILOSOPHORUM EN LA MAXIMA DOCTRINA IGNORANTIAE DE NICOLÁS DE CUSA
}

\section{THE LIBER XXIV PHILOSOPHORUM'S IMAGE OF THE INFINITA SPHAERA IN THE NICHOLAS OF CUSA'S MAXIMA DOCTRINA IGNORANTIAE}

\author{
JOSÉ GONZÁLEZ RÍOS* \\ Dr. en Filosofía \\ Universidad de Buenos Aires - Buenos Aires - Argentina \\ Consejo Nacional de Investigaciones Científicas y Técnicas \\ Instituto de Filosofía "Dr. Alejandro Korn"
}

Artículo recibido el 30 de julio de 2020; aceptado el 2I de octubre de 2020.

"josegonzalezrios@gmail.com

https://orcid.org/oooo-oooI-7537-5710

Cómo citar este artículo:

J. González. R. (2020). "La imagen de la sphaera infinita del anónimo liber xxiv philosophorum en la maxima doctrina ignorantiae de Nicolás de Cusa” en Palabra y Razón. Revista de Teología, Filosofía y Ciencias de la Religión. № I8 Diciembre 2020, pp 89-IO4 https://doi.org/IO.29035/pyr.I8.89 


\title{
RESUMEN
}

El artículo aborda el funcionamiento tanto teológico como cosmológico de la imagen de la sphaera infinita del Liber XXIV philosophorum en De docta ignorantia de Nicolás de Cusa. En primer término, se ofrece una descripción del Liber, y, de manera específica, de la Definición II. En segundo lugar, se aborda la recepción del Liber en Nicolás de Cusa, y se analiza el uso estrictamente teológico de la imagen de la sphaera infinita en De docta ignorantia I. Luego, en tercer lugar, se trata el uso cosmológico de la imagen que él realiza en De docta ignorantia II. Finalmente, en cuarto lugar, se evalúa de modo crítico el desplazamiento de la imagen de la sphaera infinita del ámbito teológico al cosmológico con la finalidad de mostrar, contra la mayoría de las interpretaciones, que el uso de la imagen en su cosmología sigue siendo estrictamente teológico.

Palabras claves: Liber XXIV philosophorum / sphaera infinita / maxima doctrina ignorantiae / metafísica / cosmología

\begin{abstract}
The present article explores the theological and cosmological aspects of the Liber XXIV philosophorum's image of the sphaera infinita in De docta ignorantia of Nicholas of Cusa. The paper is structured as follows. First, I describe the Liber, specifically focusing on its Definition II. Secondly, I address Nicholas of Cusa's reception of the Liber, analyzing the strictly theological use of the sphaera infinita's image in De docta ignorantia I. Thirdly, I study the cosmological use of said image in De docta ignorantia II. Finally, I examine the sphaera infinita's passage from the theological to the cosmological domain in the maxima doctrina ignorantiae, and against most of the hegemonic scholarship, I show that the use of the image in the Cusan cosmology remains strictly theological.
\end{abstract}

Keywords: Liber XXIV philosophorum / sphaera infinita / maxima doctrina ignorantiae / metaphysics / cosmology 


\section{I) El Liber XXIV philosophorum}

En el último tercio del siglo XII irrumpe en el occidente medieval latino un pequeño escrito de definiciones comentadas con el fin de responder a una sola pregunta: “¿Qué es Dios? Quid est Deus?”. El devenir plural de su transmisión y recepción lo volverá uno de los textos más fascinantes de la historia del pensamiento. La incertidumbre respecto de su origen, título, autor, estructura y organización integran un debate historiográfico que se extiende desde finales del siglo XIX hasta nuestros días.

El título con el que hoy conocemos este escrito, le fue conferido por el Maestro Eckhart, que tanto en su Expositio libri Genesis como en su Expositio libri Exodi lo llama Liber XXIV philosphorum². Sin embargo, en la tradición manuscrita el texto es nombrado de diversas maneras. Entre ellas: "Incipiunt uiginti quatuor proposiciones philosophorum cum commento de essencia diuina" (D), "Expliciunt uiginti quatuor diffiniciones de deo famose philosophorum uiginti quatuor gentilium famosorum" (E), "Liber de causa prima" (C) y "Diffiniciones de deo eiusdem Hermetis" (A)3. Resulta también desconocido su autor, aunque varios manuscritos lo atribuyan a Hermes Trimegisto, y lo vuelvan así un texto portador de una prisca sapientia ${ }^{4}$.

Las cuestiones referidas tanto a su autor como a su título, conducen directamente al problema del origen del Liber. En la historia de su edición, traducción y estudio se encuentran diferentes y hasta antagónicas hipótesis respecto de su procedencia. Con todo, estas diversas conjeturas pueden reducirse a dos grandes líneas de interpretación. Por una parte, la de quienes inscriben al Liber en el contexto del plural neoplatonismo del siglo XII latino. Por otra, la de Hudry, para quien el Liber procede de la Antigüedad Tardía. Según ella, el Liber podría ser la reelaboración de un texto aristotélico ${ }^{5}$, un manual de teología aristotélica redactado en la

\footnotetext{
I Como es sabido, en 1986 se dio inició al Proyecto de edición crítica y estudio del Hermes latinus, a cargo de un Equipo coordinado por Paolo Lucentini. Entre los textos que fueron editados, se encuentra el Liber viginti quattuor philosophorum. Su edición, en 1997, estuvo a cargo de Françoise Hudry, a partir de un trabajo realizado con 26 manuscritos existentes y la noticia de Io manuscritos perdidos, pero de los cuales quedan aún referencias. De aquellos 26 manuscritos, uno tan solo data del siglo XIII, diecisiete del siglo XIV y ocho del XV. Cf. F. HUDRY. Liber viginti quattuor philosophorum, Corpus Christianorum Continuatio Mediaevalis CXLIII, A. Hermes Latinus, III, Pars I. Brepols: Turnhout, 1997.

2 Echardus. Exp. Gen. 2, 2, n. 155 (LW I.2, p. I9I, I8); Echardus. Exp. Ex., I6, I8, n.9I (LW 2, p. 95, I-2). 3 Con la letra mayúscula entre paréntesis indicamos el manuscrito del Liber al que referimos, siguiendo en esto la edición crítica de Hudry. Sobre la descripción y detalle de los manuscritos, cf. F. HUDRY. Liber vignti quattuor philosophorum..., pp. L-LXXXII.
}

4 Manuscritos X, B, P, O, V, T, G, H, N, Y, Z, R.

5 Cf. F. HUDRY. Le livre des XXIV philosophes. Millon: Grenoble, 1989. 
Escuela de $\operatorname{Harran}^{6}$ o, según su última precisión, un escrito compuesto por Mario Victorino, conocido también por su Liber de definitionibus ${ }^{7}$. Sin embargo, frente a la conjetura de Hudry, nos parece decisiva en el Liber la presencia de nociones y elementos doctrinales que florecen en la atmósfera del neoplatonismo latino del siglo XII, presencia que ha sido suficientemente explorada ${ }^{8}$.

En lo que concierne a la estructura del escrito, en la edición crítica de 1997 Hudry presenta las diversas formas en que se lo transmite en los veintiséis manuscritos con los que trabaja9. Quisiéramos tan sólo referir, en función de nuestro asunto, a la forma canónica en la que aquel circuló durante la Edad Media y la Modernidad temprana, y que se conoce bajo el nombre de versio communis. Es la forma que se encuentra en los códices L, C, J, D, I, P, que traen: el Prólogo (que brinda la escena del texto), las veinticuatro definiciones y un pequeño y breve comentario que acompaña a cada una de ellas. Hudry considera, por otra parte, que ésta es la forma originaria del Liber, dado que es la que presenta el manuscrito de Laon (L), que es el más antiguo que se conserva y el único que data del siglo XIII' ${ }^{10}$.

A su vez, el Liber no presenta una organización interna explícita que establezca la relación y la concatenación entre las definiciones y sus respectivos comentarios. Con todo, de esto no se desprende que estemos en presencia de un florilegio de definiciones y comentarios tomados de

6 Cf. F. HUDRY. Liber viginti quattuor philosophorum..., p. IX y ss.

7 Cf. F. HUDRY. Le livre des XXIV philosophes: résurgence d'un texte du IVe siècle. Vrin: Paris, 2009.

8 Cf. C. BAEUMKER. "Das pseudo-hermetische 'Buch der vier- undzwanzig Meister' (Liber XXIV philosophorum). Ein Beitrag zur Geschichte des Neupythagoreismus und Neuplatonismus im Mittelalter" en Abhandlungen aus dem Gebiete der Philosophie und ihrer Geschichte. Herder: Friburgo, I9I3, pp. I7-40; D'ALVERNY. "Un témoin muet des luttes doctrinales du XIIIe siècle" en Archives d'histoire doctrinale et littéraire du Moyen Âge. $\mathrm{N}^{\circ}$ I7, 1949, pp. 223-248; W. BEIERWALTES. "Liber XXIV philosophorum" en K. RUH (ed.) Die deutsche Literatur des Mittelalters. Verfasserlexikon. De Gruyter: Berlín-Nueva York, 1985, pp. 767-770; P. LUCENTINI. Il libro dei ventiquattro filosofi. Adelphi: Milán, 1999; Z. KALUZA. "Besprechung in Liber viginti quattuor philosophorum" en Mittellateinisches Jahrbuch. $\mathrm{N}^{\circ} \mathrm{XXXV}$, 2000, pp. I6I-I66. Z. KALUZA. "Comme une branche d'amandier en fleurs. Dieu dans le Liber viginti quattor philosophorum" en P. LUCENTINI et al. (eds.). Hermetism from Late Antiquity to Humanism. Brepols: Turnhout, 2003, pp. 99-I27; K. FLASCH. Was ist Gott? Das Buch der 24 Philosophen. Beck: Múnich, 20II; A. BECCARISI. "Deus est sphaera intellectualis infinita: Eckhart interprete del Liber XXIV philosophorum" en P. TOTARO - L. VALENTE (eds.). Sphaera. Forma, immagine e metafora tra Medioevo e età moderna. Olschki: Florencia, 20I2, pp. I67-I92. Y A. BECCARISI. "Noch sint ez allez heidenischer meister wort die niht enbekanten dan in einem natiurlichen lichte? Eckhart e il Liber vigintiquattuor philosophorum" en L. STURLESE (ed.). Studi sulle fonti di Meister Eckhart II. Academic Press Fribourg: Friburgo, 20I2, pp. 73-96.

9 Cf. F. HUDRY. Liber vignti quattuor philosophorum..., pp. LXXXII-LXXXIX.

Io Cf. F. HUDRY. Liber vignti quattuor philosophorum..., p. LXI. 
fuentes no explicitadas de la tradición ${ }^{\text {II }}$. Sin embargo, el Liber mismo brinda unas pocas referencias a través de las cuales establece de modo explícito la relación entre definiciones. Y, curiosamente, esto sucede a partir de la imagen de la sphaera. El comentario a la Definición XVIII, que trae la imagen, afirma "ista sequitur ex secunda". De este modo el Liber mismo coordina las definiciones II y XVIII a partir de la metáfora de la sphaera ${ }^{12}$. Pero la imagen tiene lugar también en el comentario a la Definición XIV, que, a pesar de esto, no trae ninguna indicación que la ponga en relación con las otras dos definiciones ${ }^{13}$. Lo único que podemos desprender de esto es que el texto mismo subraya la relevancia y gravitación de la imagen de la sphaera para responder a la pregunta que orienta todo su desarrollo.

En virtud de esto, concentrémonos brevemente en la Definición II, que es objeto de nuestro análisis. Ella afirma que "Dios es una esfera infinita cuyo centro está en todas partes y su circunferencia en ninguna"14. La definición ofrece así una imagen que expresa la imposibilidad de concebir la esencia de la divinidad de modo racional (rationaliter), pues elevada al infinito, la imagen de la sphaera conduce a pensar de modo intelectual (intellectualiter) los términos de "centro" y "circunferencia"

II Hudry ha conjeturado una organización y articulación interna del escrito. En su edición de 1997, advierte tres secciones en el texto. Una primera, definiciones I-VII, en las que el Liber trataría sobre la esencia de Dios considerado en sí mismo. Una segunda, definiciones VIII-XX, en las que abordaría a Dios en relación con la creatura. Y una tercera, definiciones XXI-XXIV, en las que se consideraría a Dios en su relación con el alma humana. Cf. F. HUDRY. Liber vignti quattuor philosophorum..., pp. VII-IX. Años más tarde sugirió una nueva organización del escrito. Según ella, una primera sección trataría sobre la esencia de Dios a través del número o mónada (definiciones I, III, IV, V, VI, XII) y a través de la figura de la esfera (definiciones II, VII, XV, XVIII, XIX, XX). Una segunda, que abordaría la potencia de Dios (IX, X, XI, XIII, XIV, XXII). Y, finalmente, una tercera, la incognoscibilidad de Dios (VIII, XVI, XVII, XXI, XXIII, XXIV). Cf. F. HUDRY. Le livre des XXIV philosophes..., pp. 23-87. Es oportuno advertir que en la primera organización que ofrece, Hudry sigue un diseño que se centra en Dios, considerado en sí, en su relación con lo creado y con el alma humana. Mientras que en la segunda organización que brinda, ella parece centrarse en la comprensión humana de Dios: simbolizado por medio de las disciplinas matemáticas, considerado en su potencia y en su incognoscibilidad.

I2 La Definición II refiere a una única esfera con un único centro que está en todas partes. La Definición XVIII, en cambio, refiere a una multiplicidad de esferas y centros. Cada punto es centro de una esfera. Cf. L. VALENTE. "Sfera infinita e sfera intellegibile: immaginazione e conoscenza di Dio nel Libro dei XXIV filosofi e in Alano di Lilla" en P. TOTARO - L. VALENTE (eds.). Sphaera. Forma, immagine e metafora tra Medioevo e età moderna. Olschki: Florencia, 20I2, p. I27.

I3 Cf. F. HUDRY. Liber vignti quattuor philosophorum..., p. 2I. La Definición XIV reza: "Deus est oppositio nihil mediatione entis". Y el comentario afirma: "Haec definitio imaginari facit deum esse sphaeram in cuius centro nihil incarceratur et est continue agens sphaera divina opus divinum quo detinet nihil in suo esse aeternaliter, a quo per exuberantiam suae bonitatis vocavit in esse rem quae est quasi circa centrum. quae si ad esse actum attrahit, stabit sphaera, si ad esse possibile, redibit ad nihilum". [El subrayado es nuestro].

I4 F. HUDRY. Liber vignti quattuor philosophorum..., p. 7: "Deus est sphaera infinita cuius centrum est ubique, circumferentia nusquam".

I5 En la tradición manuscrita la sphaera infinita ha sido concebida como una sphaera intelligibilis. 
Pues, si el "centro" de la esfera está en todas partes (ubique), ya no se encuentra en un lugar localizado o determinado, sino que ocupa infinitos lugares, que son igualmente centro. En cuanto a la "circunferencia", ella no está en ninguna parte (nusquam), y, por tanto, niega todo límite o determinación para la esfera. Dios, en cuanto esfera infinita, está en todas partes y en ninguna.

La imagen, así concebida, refiere a la trascendencia y a la inmanencia de Dios. Una esfera infinita cuyo centro está en todas partes, indica la inmanencia de la divinidad, y su circunferencia en ninguna, simboliza la trascendencia de la divinidad, que por nada es determinada o limitada. El breve comentario que sigue a la definición, subraya esta consideración respecto de la inmanencia y la trascendencia de Dios al afirmar que la definición hace imaginar la vida (vita) de la causa primera (prima causa) como un continuum de vida ${ }^{\mathrm{I}}$. Una vida infinita que está en todas partes y en ninguna.

\section{2) La imagen de la sphaera infinita en la consideración de lo máximo en sentido absoluto o Dios en De docta ignorantia I de Nicolás de Cusa}

Como ha mostrado Lucentini, el Liber XXIV philosophorum y, sobre todo, la imagen de la sphaera infinita de la Definición II, han tenido una recepción plural a partir de su irrupción en el occidente medieval latino ${ }^{17}$. Pensadores de tradiciones diversas lo han acogido como una autoridad pagana en la elaboración de sus respectivos sistemas de pensamiento. Con todo, su asimilación ha sido notaria en la tradición neoplatónica. Sturlese ha mostrado la presencia de textos herméticos y del Liber en particular, en la obra de Alberto Magno ${ }^{18}$. Por su parte, Sannino ha estudiado la presencia del Liber en Bertoldo de Moosburg ${ }^{19}$. Y Beccarisi ${ }^{20}$,

\footnotetext{
Cf. A. BECCARISI. "Noch sint ez allez heidenischer meister wort..., pp. 77 y ss.

I6 El concepto de continuum es relevante en el Liber, especialmente en las definiciones I, II, IV y XIV.

I7 P. LUCENTINI. "La esfera infinita e la fortuna della seconda proposizione del Libro dei XXIV filosofi nel Medioevo", en P. Totaro - L. Valente (eds.). Sphaera. Forma, immagine e metafora tra Medioevo ed Età Moderna. Olschki Editore: Firenze, 20I2, pp. I-II; P. LUCENTINI. Il libro dei ventiquattro filosofi, Adelphi: Milán, 1999, pp. I05-150.

I8 Cf. L. STURLESE. "Saints et magiciens: Albert le Grand en face d'Hermès Trismégiste" en Archives de Philosophie. $\mathrm{N}^{\circ}$ 43, 1980, pp. 615-634. Y cf. L. STURLESE. "Proclo e Ermete in Germania da Alberto Magno a Bertoldo di Moosburg" en K. FLASCH (ed.). Von Meister Dietrich zu Meister Eckhart. Meiner: Hamburgo, 1984, pp. 22-33.

I9 Cf. A. SANNINO. "Il Liber viginti quattuor philosophorum nella metafisica di Bertoldo di Moosburg" en A. BECCARISI - R. IMBACH - P. PORRO (eds.). Per perscrutationem philosophicam. Neue Perspektiven der mittelalterlichen Forschung. Meiner: Hamburgo, 2008, pp. 252-272.

20 Cf. A. BECCARISI. "Deus est sphaera intellectualis infinita...”, pp. I67-192. Y cf. A. BECCARISI.

"Noch sint ez allez heidenischer meister wort...", pp. 73-96.
} 
su circulación y fuerza en Eckhart, tanto en la obra latina como en la alemana. Todas éstas, por cierto, fuentes explícitas de Nicolás de Cusa ${ }^{21}$.

Hallauer $^{22}$, Arfée $^{23}$, Federici Vescovini ${ }^{24}$ y D'Amico ${ }^{25}$ han estudiado la recepción de textos herméticos en la génesis y en el desarrollo de la maxima doctrina ignorantiae. Entre ellos, el Asclepius (a través del Codex Bruxellensis que se encuentra en la Blibliotheque Royale Albert Ier I0054-56), que Nicolás de Cusa anota con marginales que fueron editadas por Arfé en Cusanus Texte III. Marginalien 5. En cuanto a la lectura y estudio del Liber, si bien no se conoce con certeza qué manuscrito leyó el Cusano, a partir de las menciones explícitas que hace de él, puede conjeturarse que trató con un códice que traía la versio communis ${ }^{26}$.

En una primera aproximación, encontramos un "aire de familia" entre el Liber y ciertos postulados de la maxima doctrina ignorantiae: la comprensión de la unitrinidad de Dios a través del concepto de monas, que el Liber presenta en la Definición I ${ }^{27}$; la inmanencia y la trascendencia del principio, que se expresa, como advertíamos, en la imagen de la sphaera infinita que trae la Definición II; la consideración de Dios como aquello mayor que lo cual nada puede ser pensado, que el Cusano recupera en su definición de lo maximum, en términos del ser y no del pensar como

2I Es necesario advertir, en este sentido, que el contacto del Cusano con el Liber también ha estado mediado por estas fuentes de la tradición de la que él mismo se sabe heredero. Cf. J. GONZÁLEZ RÍOS. "A propósito de una fuente de los pensadores de la Escuela Renana: el anónimo Liber viginti quattuor philosophorum" en Revista Principios. Vol. 2I/ Nº 37, 20I5, pp. 99-I2I.

22 Cf. H. HALLAUER. "Cod. Harl 363I ubd 3915" en Mitteilungen und Forschungsbeiträge der Cusanus-Gesellschaft. N Io, I973, pp. 94-I03.

${ }_{23}$ Cf. P. ARFÉ. "Ermete Trismegisto e Nicola Cusano" en P. LUCENTINI - I. PARRI - V. PERRONE COMPAGNI (eds.). Hermetism from Late Antiquity to Humanism. La tradizione hermetica dal mondo tardo-antico all' Umanesimo. Brepols: Turnhout (Instrumenta Patristica et Mediaevalia, 40), 2003, pp. 223-243.

24 Cf. G. FEDERICI VESCOVINI. "Les métamorphoses de quelques propositions hermetiques après le De docta ignorantia (I440)" en H. PASQUÁ (ed.). Identité et différence dans l'oeuvre de Nicolas de Cues (I4OI-I464). Éditions Peeters: Walpole, 20II, pp. I-I4.

25 Cf. C. D'AMICO. "Nicolás de Cusa: lector del Asclepius y del Liber XXIV philosophorum" en C. D’AMICO - V. BUFFON (eds.). Hermes Platonicus: La recepción del hermetismo vinculado con el platonismo en la filosofía medieval y de la Modernidad temprana. UNR: Rosario, 2015, pp. I46-I59.

26 Cf. G. FEDERICI VESCOVINI. “Les métamorphoses de quelques propositions hermetiques...", pp. I-I4.

27 La Definición I, según la cual "Deus est monas monadem gignens, in se unum reflectens ardorem", expresa desde un punto de vista estrictamente filosófico, el dinamismo intratrinitario de lo divino. Indica, por medio de la repetición del concepto de monas, el repliegue de lo divino en sí mismo sin multiplicación, y, por tanto, sin alteridad. Esto mismo subraya el comentario a la definición, que afirma que esta multiplicación numérica del uno no implica multiplicidad (multitudine). D’Amico ha mostrado que la presencia de la Definición I del Liber en De docta ignorantia I c.8 se encuentra mediada por una de las interpretaciones que Eckhart ofrece de la fórmula "ego sum qui sum" en su Comentario al Éxodo: la mónada (sum) que engendra su misma igualdad (sum), unidas de modo eterno (qui). Cf. Echardus. In libri Exodi III I4, n. I6 (LW II, p. 2I, I et sqq.). Cf. C. D’AMICO. "Nicolás de Cusa: lector del Asclepius...", pp. I52-I53. 
en el caso de la Definición V del Liber ${ }^{28}$; la abundancia y suficiencia del principio, que está por sobre lo que es, conforme lo indica la Definición $\mathrm{XI}^{29}$; la consideración de que Dios es el único que vive en la intelección de sí mismo, según la Definición XX; y la vía de la negación, que, entre otros, tiene lugar en la Definición XVI, al afirmar que Dios es lo único que las palabras no pueden significar con precisión ni las mentes entender. Finalmente, la Definición XXIII "Dios es aquel que es conocido por la mente solo a través de la ignorancia" (Deus est qui sola ignorantia mente cognoscitur), que expresa el corazón mismo de la máxima doctrina de la ignorancia, que Nicolás de Cusa sintetiza en el título del Capítulo i del Libro I de De docta ignorantia: "De qué modo saber es ignorar" (Quomodo scire est ignorare).

Respecto de esta afinidad entre ambos no es menor advertir que el manuscrito de Erfurt (Kartause Salvatorberg C. 27), nombra las definiciones del Liber como "enigmaticae"30. De este modo, el Liber fue también interpretado en la tradición manuscrita como un compendium de enigmas, esto es, imágenes que vuelven visible lo invisible de modo intelectual a través de definiciones. Conforme a la doctrina ignorante, podríamos decir que las definiciones del Liber resquebrajan la esfera de la ratio y guían la mente hacia la esfera del intellectus. Por eso, el breve comentario que las acompaña busca traducir la fuerza intelectual de aquellas en el ámbito de un discurso y comprensión racional ${ }^{31}$.

Pero más allá de este "aire de familia”, se trata, como indicábamos, de una fuente que el Cusano cita de modo explícito, a veces a través de la mediación de otras fuentes caras a su doctrina, como en el caso de Eckhart. En lo que sigue quisiéramos referir exclusivamente al empleo que hace de la imagen de la sphaera infinita de la Definición II del Liber en De docta ignorantia, tanto al tratar sobre lo máximo en sentido absoluto o Dios en el Libro I, como al abordar una segunda consideración de lo máximo, en cuanto contracto o universo en el Libro II, en el marco de

28 Para las referencias a las obras de Nicolás de Cusa seguimos la edición crítica de la Nicolai de Cusa, Opera Omnia, iussu et auctoritate Academiae Litterarum Heidelbergensis ad codicum fidem edita (h). Ofrecemos la abreviatura canónica del texto, el libro y capítulo cuando corresponda y entre paréntesis el tomo de la edición crítica y el parágrafo correspondiente. Cf. De doc. ig. I c.2 (h I n.4): "Maximum autem hoc dico, quo nihil maius esse potest".

29 Se sostiene con ello que lo que es, en virtud de su determinación, excluye todo aquello que no es. Con todo, Dios no excluye nada de sí, sino que todo lo contiene y conserva pues "superest qui non clauditur". La relación esse-superesse, que juega en la Definición XI, expresa una comprensión de la teología negativa que Nicolás de Cusa practica en el conjunto de su obra.

30 Cf. F. HUDRY. Liber vignti quattuor philosophorum..., p. LXXXI: "Libellus de diffinicionibus Dei philosophorum et theologorum et ponuntur ibidem 23 diffiniciones enigmatice Dei."

3I I. PARRI. "Note sul Libro dei ventiquattro filosofi" en S. CAROTI - R. PINZANI (eds.). "Ob rogatum meorum sociorum": studi in memoria di Lorenzo Pozzi. Angeli: Milán, 20oo, p. 156. 
una cosmología audaz que contiene tesis que "para muchos resultan insólitas", como él mismo lo afirma en la Carta con la que cierra la obra ${ }^{32}$.

El Cusano recupera la imagen de la sphaera infinita referida a Dios, considerado en sí mismo, conforme a una plural tradición teológica de la que se sabe heredero ${ }^{33}$. Así, en De docta ignorantia I al considerar lo máximo absoluto en su unitrinidad, emplea la imagen en el contexto de la transsumptiva proportione que practica a partir del Capítulo II. Sin mencionar al Liber ni citar la definición de modo completo, Nicolás traspone la imagen de la sphaera infinita a la actualidad infinita de Dios ${ }^{34}$. Pues con la línea infinita, él refiere a la rectitud absoluta, con el triángulo infinito, a la trinidad absoluta, con el círculo infinito, a la unidad absoluta y con la esfera infinita, a la existencia absolutamente en acto de lo máximo (actualissima Dei existentia). Así, referida a Dios considerado en sí mismo, la imagen de la sphaera infinita, que corona, como indicamos, la serie de figuras geométricas (línea, triángulo, círculo, esfera), resulta una manuductio que permite comprender intellectualiter que Dios es la actual perfección de todo, y, por esto, vida de todas las cosas. No podemos dejar de enfatizar aquí la pregnancia del concepto de vita en el Liber, y no en último término en el contexto del comentario a la Definición II. La esfera infinita (que es Dios) ha de ser pensada como vida de todas las cosas. En términos cusanos, como todo posible ser en acto $^{35}$.

\section{3) La imagen de la sphaera infinita en la consideración de lo máximo en sentido contracto o universo en De docta ignorantia II de Nicolás de Cusa}

La imagen de la Definición II del Liber vuelve a irrumpir, y de modo completo, en De docta ignorantia II, donde el Cusano trata sobre lo máximo en una segunda consideración como contracto o universo, esto

32 Cf. De doc. ig. III Ep. (h I n.264): "Secundus ex illo pauca de universo supra philosophorum comunem viam elicit rara multis".

33 Alanus de Insulis. Regulae, 7, ed. Haring, pp. 13I-32; Alanus de Insulis. Sermo de sphaera intelligibili, ed. M.-T. d'Alverny, Alain de Lille. Textes inédits. Vrin: Paris, 1965, pp. 297-306. Cf. Echardus. Lectio I super Ecclesiastici c. 24, 23, nn. 17-20 (LW 2, pp. 246-48); Echardus. Expositio lilni Exodi, I6, I8, n. 9I (LW 2, pp. 94-95); et passim.; Bertoldo di Moosburg. Expositio, prop. 98 C, Cod. Vat. Lat. 2192, f. $154 \mathrm{vb}$.

34 De doc. ig. I c. I9 (h I n.56): "Insuper, sicut maxima linea non plus est linea, triangulus, circulus vel sphaera, sed in veritate est illa omnia absque compositione, ut ostensum est: ita consimiliter maximum simpliciter est ut maximum lineale, quod possumus dicere essentiam; est ut triangulare, et potest dici trinitas; est ut circulare, et potest dici unitas; est ut sphaerale, et potest dici actualis existentia".

35 De doc. ig. I c.2 (h I n.4): "tunc est actu omne possibile esse, nihil a rebus contrahens, a quo omnia". 
es, como la unidad de lo diverso (unus-versus) ${ }^{36}$. Esta unidad contracta o esfera que es el universo, procede de lo máximo absoluto o esfera absoluta. Sin embargo, para Nicolás de Cusa es una esfera inmanente a la multiplicidad, y no subsiste separada de ella. Es, por tanto, máxima, en la medida en que procede de lo máximo absoluto, pero, a su vez, contracta, pues no perdura separada de la multiplicidad. Con esta distinción entre una unidad o esfera absoluta y una contracta el Cusano no postula una diferencia sustancial. Se trata, en todo caso, de consideraciones diversas de lo máximo, unidad o esfera.

La clave de esta segunda consideración de lo máximo -como universo- se cifra en el concepto de contractio. Esta noción debe entenderse, en primer lugar, como no-absoluto. En segundo lugar, como $a b$ alio o a causa, al establecerse la distinción entre lo que es a partir de sí (a se) y lo que tiene su procedencia a partir de lo que es a se. En tercer lugar, indica el modo propio del despliegue de Dios o explicatio dei en las cosas. El universo expresa, en tanto máximo contracto, la primera unidad contracta de lo real. Desde este punto de vista, es lo más cercano a lo absoluto en cuanto a grados de contracción.

Con esta consideración de lo máximo en cuanto universo, Nicolás de Cusa tematiza el paso de lo infinito a lo finito ${ }^{37}$. Lo máximo contracto opera como la mediación que permite explicar el pasaje de la unidad absoluta a la cosa singular ${ }^{38}$. Pues en lo máximo absoluto todo se encuentra complicadamente (complicative) de modo idéntico e indiferenciado. A su vez, en su despliegue o explicación (explicatio) como multiplicidad, las cosas preservan su unidad. Esa instancia de unidad en las cosas (quidditas contracta) es lo que el Cusano entiende por universo: un principio metafísico que unifica la multiplicidad en tanto múltiple, ${ }^{39}$ y no subsiste, como advertíamos, separado de ella.

36 De doc. ig. II c.I2 (h I n.I62).

37 Cf. De doc. ig. II c.4 (h I n.II7).

38 De doc. ig. II c.4 (h I n.II6): "Et ita intelligi poterit, quomodo Deus, qui est unitas simplicissima, existendo in uno universo est quasi ex consequenti mediante universo in omnibus, et pluralitas rerum mediante uno universo in Deo".

39 Cf. De doc. ig. II c.4 (h I n.II3). Respecto de la ontología de lo creado, baste al menos indicar que para Nicolás de Cusa cada cosa posee no solo una esencia absoluta (quidditas absoluta), que es complicación absoluta de todo, sino también una esencia contracta (quidditas contracta), que expresa su unidad en la alteridad. Cada cosa posee una naturaleza, que en sentido absoluto la hace una e indiferenciada con todo y en sentido contracto la identifica como otra respecto de las demás cosas. Así, en la unidad infinita y absoluta de Dios, todas las cosas están complicadas indiferenciadamente. En la unidad indeterminada e ilimitada del universo, las cosas están unificadas en su diversidad. Lo que se explicita en la diversidad de las cosas es el universo todo, tal como lo advierte el título del Capítulo 5 de este Libro Segundo: "Quodlibet in quolibet". 
El Cusano establece así la distinción entre lo maximum concebido a se, y, por tanto, absoluto, y lo maximum considerado como ab alio, esto es, como contracto. Aquello que encuentra su principio en sí, él lo define como infinito negativamente (negative), en la medida en que se niega de él cualquier contracción o determinación. Como hemos indicado, es todo lo que puede ser, pura potencia en acto. En él prevalece la potencia por sobre la actualidad, que implica necesariamente determinación. Sin embargo, el universo es definido también como infinito, pero en un sentido muy particular, como privativo (privative) ${ }^{40}$. De aquí que el universo, tal como lo concibe Nicolás de Cusa, no es absolutamente infinito o infinito negativo, pero tampoco es finito, como las cosas singulares. Es infinito en tanto que ilimitado, pues no se encuentra determinado por ninguna cosa en acto, sólo por lo máximo en sentido absoluto.

El universo es, en este sentido, esfera infinita de modo privativo o contracto. Su potencia no puede actualizarse totalmente, ya que de ese modo devendría en absoluto. En virtud de ello, lo único que excede a lo máximo en tanto contracto o universo es lo máximo en sentido absoluto, donde toda potencia está actualizada. El universo no puede ser mayor de lo que es, puesto que la materia o possibilitas essendi que lo constituye no se extiende más allá de sí. A partir de la noción de potencia, se desprende entonces una distinción entre la potencia de la esfera considerada como absoluta y la potencia de la esfera en cuanto contracta o universo. ${ }^{41}$

De la concepción metafísica de lo máximo en sentido contracto o universo, como aquella esfera en la que, en virtud de la pluralidad, hay grados de menor y mayor (minus et magis), se sigue que no puede haber, en su diferencia, dos cosas que sean absolutamente idénticas. En su explicación o despliegue, las cosas que componen el universo expresan variaciones de grado, de más y de menos, en cuanto al acto y la potencia. Del mismo modo, no puede darse en el universo un movimiento que sea máximo y mínimo, ya que por el principio de la coincidencia de los opuestos (coincidentia oppositorum) un movimiento (motus) máximo coincide con un movimiento mínimo, y, por tanto, con la quietud (quies). Así, puesto que no se da en el universo un movimiento máximo y mínimo a la vez, todos los astros que lo componen, entre ellos la Tierra y las llamadas "estrellas fijas" deben moverse, según grados, un más y un

40 De doc. ig. II c.I (h I n.97).

4I De doc. ig. II c.I (h I n.97): "Quare, licet; in respectu infinitae Dei potentiae, quae est interminabilis, universum posset esse maius: tamen resistente possibilitate essendi aut materia, quae in infinitum non est actu extendibilis, universum maius esse nequit; et ita interminatum, cum actu maius eo dabile non sit, ad quod terminetur; et sic privative infinitum". 
menos (magis et minus), que se da entre el máximo y mínimo movimiento absoluto.

Ahora bien, puesto que no puede haber un movimiento máximo y mínimo a la vez, tampoco puede haber un centro físico absoluto, esto es, máximo y mínimo a la vez. Y dado que no puede haber un centro físico absoluto, tampoco puede haber una circunferencia o límite. Por este motivo, es imposible que el universo o mundo (mundus) tenga un centro fijo e inmóvil. Pues si tuviese un centro fijo y, por tanto, una circunferencia, tendría un límite, más allá del cual habría algo ${ }^{42}$. Así como la Tierra no es el centro del mundo, tampoco la esfera de las estrellas fijas es su circunferencia ${ }^{43}$. En todo caso hay un único centro metafísico: Dios (coincidentia oppositorum); que es centro de la Tierra, de las esferas fijas y de todo lo que compone el universo. Y así como Dios es centro absoluto, es, a su vez, circunferencia absoluta. Es, en sentido, la esfera infinita absoluta en la que coinciden centro y circunferencia ${ }^{44}$.

Pero muchos creen, a partir de las mediciones y proporciones que practican por medio de las artes con las que estudian el universo, especialmente, las del quadrivium, estar en el centro de la esfera del universo. Frente a esto, la maxima doctrina ignorantiae enseña que el centro y circunferencia de la esfera del universo no pueden ser comprendido de modo preciso $^{45}$. Y citando entonces la Definición II del Liber en el Capítulo I2 el Cusano afirma que: "la máquina del mundo tendrá el centro en cualquier parte y la circunferencia en ninguna, porque su circunferencia y su centro son Dios" ${ }^{46}$, que es todo en todo y nada de todo.

\section{Consideración Final}

42 Cf. De doc. ig. II c.II (h I n.I56): "Centrum igitur mundi coincidit cum circumferentia. Non habet igitur mundus circumferentiam. Nam si centrum haberet, haberet et circumferentiam, et sic intra se haberet suum initium et finem, et esset ad aliquid aliud ipse mundus terminatus, et extra mundum esset aliud et locus; quae omnia veritate carent".

43 De doc. ig. II c.II (h I n.I57): "Nam si esset etiam distanter a centro et circa axim per polos transeuntem, ita quod una parte esset elevata versus unum polum, in alia depressa versus alium, tunc hominibus tantum a polis distantibus, sicut horizon se extendit, sola medietas sphaerae appareret, ut est manifestum".

44 Cf. De doc. ig. II c.II (h I n.I57): "Qui igitur est centrum mundi, scilicet Deus benedictus, ille est centrum terrae et omnium sphaerarum atque omnium, quae in mundo sunt; qui est simul omnium circumferentia infinita".

45 Cf. De doc. ig. II c.II (h I n.I6I).

46 De doc. ig. II, c.I2 (h I n.I62): "Unde erit machina mundi quasi habens undique centrum et nullibi circumferentiam, quoniam eius circumferentia et centrum est Deus, qui est undique et nullibi”. 
Como hemos mostrado, Nicolás de Cusa resulta heredero de una tradición que, conforme a la Definición II del Liber, identificó a Dios, considerado en sí mismo, con una sphaera infinita. Sin embargo, en la máxima doctrina de la ignorancia, el Cusano realiza el desplazamiento de la imagen de la esfera infinita del ámbito teológico al cosmológico, al considerar la vida de lo máximo en sentido contracto como universo o máquina del mundo. Este desplazamiento sin duda resultó novedoso y ciertamente inaudito, como lo han puesto de manifiesto diversos investigadores ${ }^{47}$. Recuperando el emblemático título de la obra de Koyré, auspició el paso del mundo cerrado al universo infinito. Así muchos han visto en la cosmología cusana el umbral de una nueva época, la del copernicanismo.

En este sentido, podemos advertir el efecto del uso cosmológico de la imagen de la esfera infinita que hace el Cusano apelando a uno de sus más notorios lectores en el contexto de la Modernidad temprana. En ocasión de la fundamentación metafísica del copernicanismo, Giordano Bruno en el Diálogo V de su escrito De la causa, principio e uno (1583), cita la Definición II del Liber, secularizándola y proclamando el autoagotamiento de lo divino en el universo infinito. Más aún, en su remisión tiene presente no sólo aquella definición del Liber sino también el uso que el Cusano ha hecho de ella, tal como mostramos, en De docta ignorantia $\mathrm{II}^{48}$.

47 Cf. D. MAHNKE. Unendliche Sphäre und Allmittelpunkt, Beiträge zur Genealogie der mathematischen Mystik. M. Niemeyer: Halle, I937; A. KOYRÉ. From the closed world to the infinite universe. Johns Hopkins Press: Baltimore, 1957, pp.5-27; K. HARRIES. "The Infinite Sphere: Comments on the History of a Metaphor" en Journal of the History of Philosophy. $\mathrm{N}^{\circ}{ }^{\circ} 3$, I975, pp. 5-I5; E. BRIANT. "Transitions to a Modern Cosmology: Meister Eckhart and Nicholas of Cusa on the Intensive Infinite." Journal of the History of Philosophy, Volume 37, 4 (1999), 575-600; P. LUCENTINI. "La esfera infinita e la fortuna della seconda proposizione del Libro dei XXIV...", p. IO-II; SECCHI. "Declinazioni della sfera in Niccolò Cusano" en P. TOTARO - L. VALENTE (eds.). Sphaera. Forma, immagine, metafora tra Medioevo ed età moderna. Olschki: Florencia, 2015, pp. 245-260.

48 G. BRUNO. De la causa, principio et uo. Einaudi: Torino, 1973, A I45: "Se il punto non differisce dal corpo, il centro da la circonferenza, il finito da l'infinito, il massimo dal minimo: sicuramente possiamo affirmare che l'universo è tutto centro, o che il centro de l'universo è per tutto: e che la circunferenza non è in parte alcuna, per quanto è differente dal centro; o pur che la circonferenza è per tutto, ma il centro non si trova in quanto che è differente da quella. Ecco come non è impossibile, ma necessario che l'ottimo, massimo, incomprehensibile, è tutto, è per tutto, è in tutto, perché come semplice et indivisibile può esser tutto, esser per tutto, essere in tutto". En lo que concierne a la asimilación productiva que realiza el Nolano del anónimo Liber XXIV philosophorum, la mayoría de los intérpretes circunscriben su presencia a la Definición II del Liber, que emplea en su escrito De la causa, principio e uno (Dialogo V: 32I). Con todo, esta misma definición aparece en obras como el Acrotismus (Liber I, Articulus IV: 98), Articuli adversus mathematicos (T V: I4), De monade (c. II: 342) y De immenso (Liber II, IX: 99). Pero la presencia de este breve, potente y enigmático Liber se hace realmente notoria en la Summa terminorum metaphysicorum (T V, Praxis descensus "De Deo"). Allí Bruno refiere no sólo a la segunda definición, sino que extiende su consideración a las definiciones IV, V, VI, VII, VIII, X, XVI, XVII, XIX, XXI, XXIV, que aparecen en entradas como "veritas", "principium", "intellectus", "cognitio", "voluntas", "actio", "finis", "nomen", entre otros. De aquí que uno de los objetivos del capítulo sea explorar la 
Sin embargo, aun cuando el Cusano realiza el traspaso de la imagen de la sphaera infinita del ámbito teológico al cosmológico en la máxima doctrina ignorantiae, y con esto auspicia, de cierto modo, el horizonte de la concepción metafísica de un universo infinito, con todo, el uso cosmológico de la imagen, como hemos mostrado, es aun teológico, y encuentra su fundamento en la consideración de lo máximo en sentido absoluto: Dios es centro y circunferencia absoluto; tal como se lee en la cita de la Definición II del Liber que Nicolás de Cusa presenta en De docta ignorantia II.

Por eso, para concluir, podemos apoyar la interpretación que ofrecemos apelando a otro escrito cusano. Se trata de una obra de senectud en la que Nicolás de Cusa recupera el fundamento teológico de su cosmología. Un año antes de morir, él culmina en Roma la redacción de los dos libros que componen De ludo globi (I463). En el Libro II explica el sentido místico (mystica sententia) del juego de las esferas que allí propone ${ }^{49}$. Empleando el ojo de la mente (oculus mentis), advierte, podemos ver que nada es mayor o menor (maius aut minus) que lo que está en todas las $\operatorname{cosas}^{50}$. De aquí que aquello que está en todas las cosas sea el máximo y mínimo (maximum et minimum) ejemplar de todas, como sucede en el caso de los números: cada número es un número porque la unidad (unitas) está en él ${ }^{5 !}$.

En aquel contexto el ejemplar de todas las cosas es identificado por el Cusano con el concepto de vida (vita), una noción cara, como hemos indicado, tanto a su propio sistema de pensamiento como al Liber XXIV philosophorum ${ }^{52}$. La vida está en todas las cosas, como un centro que está en todas partes. Esto le da la ocasión a Nicolás para volver, aunque sea de modo parcial, sobre la imagen de la esfera infinita, reforzando de este modo el carácter estrictamente teológico de su cosmología, pues concluye allí: "Y advierte luego el dictum del sabio que dijo que Dios es un círculo cuyo centro está en todas partes [...], así Dios está en todo"53.

presencia y funcionamiento del Liber en el archipiélago de la filosofía nolana. Cf. S. MANCINI, La sfera infinita. Identità e differenza nel pensiero di Giordano Bruno, Milano: Mimesis, 2000.

49 Cf. De ludo globi, II (h IX n.6I-62).

50 Cf. De ludo globi, II (h IX n.62).

5I Cf. De ludo globi, II (h IX n.63).

52 Cf. De ludo globi, II (h IX n.69 et sqq.)

53 De ludo globi II (h IX n.84): "Et postquam advertis dictum sapientis, qui aiebat deum circulum, cuius centrum est undique [...] ita deus in omnibus". Es necesario notar que el Cusano usa el concepto bidimensional de círculo y no el tridimensional de esfera, dado que se encuentra explicando allí la estructura del tablero del juego de las esferas a partir del cual, como una manuductio, lleva adelante su especulación. 
cc) (1) Esta obra está bajo una licencia de Creative Commons cC) $\mathrm{EY}$ NC SA Reconocimiento-No-Comercial-Compartir Igual 4.0 Internacional. 
